\title{
MANEJO CLÍNICO DE UNA FRACTURA COMPLICADA DE CORONA: REPORTE DE UN CASO
}

\author{
Claudia Milena Riveros Alejo. \\ Odontöloga, U. El Bosque, Especialista en Gerencia Hospitalaria, Escuela Superior de \\ Administraciön Pública. Especialista en Relaciones Jaborales, U. Autónoma de Bucaramanga. \\ Residente de I ario, Especialización en Odontopediatria, U. Santo Tomós
}

Auror responsable de correspondencia: Claudia Milena Riveros A. Correo electrónico: milevale_odontopediatria@yaboo.es.

\begin{abstract}
RESUMEN
El trauma dental es una situación muy común en niños y adolescentes. Se presenta con frecuencia entre los 7 los 10 años de edad. Dentro de estas lesiones traumáticas se encuentra la fractura complicada de la corona que afecta en la mayoria de los casos a los incisivos centrales superiores. El propósito de este reporte de caso es mostrar la reposición del fragmento dental en una paciente que sufrió una fractura complicada de la corona. [Riveros CM. Manejo clinico de una fractura complicada de la corona: reporte de un caso. Ustasalud Odontología 2005; 4: 59 - 64]
\end{abstract}

Palabras clave: Fractura coronal complicada, Fragmento dental, Lesiones traumáticas.

\section{CLINICAL TREATMENT OF A COMPLICATED CROWN FRACTURE: REPORT OF A CASE}

\begin{abstract}
Dental trauma is a common event among children and adolescents. Frequently, it occurs in ages between the 7 and 10 years of age. Within these traumatic injuries is the complicated crown fracture that affects in most of the cases the superior central incisors. The purpose of this paper is to show the reattachment of the dental fragment in a patient who underwent this traumatic injury.
\end{abstract}

Key words: Complicated crown fracture. Tooth fragment, Traumatic injuries, Reattachment.

Recibido para publicación: 10 de mureo de 2005. Aceptado para publicación: 25 de mayo de 2005.

\section{INTRODUCCIÓN}

De acuerdo con el III Estudio Nacional de Salud Bucal, en Colombia un $16 \%$ de las urgencias Odontológicas corresponden a fracturas dentales causadas por traumatismos. ${ }^{.}$A los 12 años de edad un 12 a 33\% de los niños y un 4 a $19 \%$ de las niñas han padecido algún tipo de trauma. Los niños son afectados dos veces más que las niñas tanto en la dentición primaria como en la permanente. ${ }^{2.5}$

La mayoría de los traumas dentales afectan los dientes anteriores, especialmente los incisivos centrales superiores, seguidos por los insicivos centrales inferiores $e$ insicivos laterales superiores. ${ }^{1}$

\section{Etiologia y factores pedisponentes}

En la dentición permanente, el trauma dentoalveolar sucede entre los 7 y 10 años; generalmente, es causado por caídas o colisiones mientras los niños juegan y corren; aunque también se destacan los accidentes en bicicleta como un factor causal frecuente. ${ }^{2}$

Estas lesiones pueden ser el resultado de traumas indirectos o directos. El trauma indirecto se presenta cuando el arco dental inferior es enérgicamente cerrado contra el superior; favorece las fracturas coronales o de coronaraíz en la región de premolares y molares, así como la posibilidad de fracturas mandibulares en la región condilar y de la sínfisis. El trauma directo se da cuando el 
diente en sí mismo es golpeado e implica afección en la región anterior. La sobremordida horizontal aumentada con protrusión de incisivos superiores y ausencia de selle labial son importantes factores predisponentes para este tipo de trauma. ${ }^{2.6}$

Otra forma de trauma en niños se produce por maltrato infantil, un $50 \%$ de estos niños presentan lesiones orofaciales. Esta forma de violencia es recurrente y cada vez más grave. Las consecuencias fisicas y psicológicas son numerosas e incluyen, incapacidad, lesiones como laceraciones, fracturas, quemaduras, contusiones, daño cerebral e inclusive la muerte. Se desarrollan múltiples problemas en el desarrollo social y afectivo exhibiendo una mayor probabilidad de manifestar comportamientos delincuenciales y violentos. ${ }^{7.8}$

Las injurias traumáticas con fractura de un incisivo permanente son una experiencia desagradable para el paciente joven y constituyen una causa importante de tratamiento endodóntico en niños. Hay que restablecer lo antes posible la estética del joven que ha sufrido un traumatismo oral, para evitar que tenga problemas por sentirse diferente a los demás. Slack y Jones en 1995, observaron que los traumatismos dentales con fracturas y desfiguración influían negativamente en las actividades escolares y en la conducta de estos niños. ${ }^{4}$

\section{Clasificación}

La clasificación de las lesiones traumáticas dentales proporcionada por Andreasen \& Andreasen está basada en un sistema adoptado por La Organización Mundial de la Salud (OMS) e incluye las siguientes lesiones:

\subsection{Injurias a los Tejidos Dentales Duros y a la Pulpa:}

2.1.1 Infracción del Esmalte.

2.1.2 Fractura del Esmalte.

2.1.3 Fractura de Esmalte y Dentina.

2.1.4 Fractura Complicada de Corona.

2.2 Injurias a los Tejidos Dentales Duros, a la Pulpa y al Proceso Alveolar:

2.2.1 Fractura de la Corona y Raiz.

2.2.2 Fractura de la Raíz.

2.2.3 Fractura Alveolar.

2.3 Injurias a los Tejidos Periodontales:

2.3.1 Concusión.

2.3.2 Subluxación.

\subsubsection{Luxaciones:}

2.3.4.1 Luxación Lateral.

2.3.4.2 Luxación Intrusiva.

2.3.4.3 Luxación Extrusiva. 2.3.5 Avulsión. ${ }^{9}$

\section{Complicaciones y pronóstico}

El trauma dentoalveolar causa hiperemia de la pulpa, cuya intensidad no siempre se puede determinar con los métodos de diagnóstico disponibles. La congestión y la alteración del flujo sanguíneo en la pulpa provocan alteraciones degenerativas irreversibles que, después de cierto tiempo, generan necrosis de la pulpa dental. ${ }^{1{ }^{110}}$

Feiglin, en 1996, estudió la respuesta dental a injurias traumáticas; sugiere que los episodios de trauma tienen diferentes pronósticos, algunos desfavorables, otros moderados y en algunos casos excelentes. Esto depende del tipo de trauma y de la edad del paciente, entre otros factores. Además corroboró que la reacción de la pulpa dental a un trauma puede ser extremadamente variable, y va desde una necrosis inmediata hasta una calcificación a largo plazo. ${ }^{10.11}$ Por lo tanto es absolutamente esencial monitorear continuamente dientes traumatizados, mediante pruebas pulpares y radiografias, este monitoreo puede ser por cinco años y más frecuentemente después del trauma para asi detectar cambios adversos y tratarlos tan pronto como ellos ocurren. ${ }^{24.610}$

Por otra parte, si se decide aplazar la restauración debido a la poca fiabilidad del pronóstico de la pulpa, se puede desarrollar un cuadro de maloclusión en pocos días ocasionado por la ausencia del contacto proximal normal con los dientes adyacentes, quienes modificarán su posición, con su borde incisal dirigido hacia la zona donde estaba la estructura dental que se perdió. Posteriormente ésta pérdida de espacio puede constituir un problema para la restauración final. ${ }^{2}$

4. Fractura complicada de corona - Guia de Manejo Este tipo de fractura involucra esmalte, dentina y pulpa. La tabla 1 describe el protocolo de manejo sugerido por la Asociación Internacional de Traumatología Dental en el 2001."

La mayor preocupación después de una exposición pulpar en un diente inmaduro es la preservación de la vitalidad pulpar para continuar con el proceso de formación radicular y el cierre apical. La pulpa expuesta debe ser sellada para impedir la contaminación, se ha demostrado que la ausencia de infección es necesaria para la reparación pulpar. .4.10.11.14 $^{2}$ 


\begin{tabular}{|l|l|}
\hline \multicolumn{1}{|c|}{$\begin{array}{c}\text { Fractura Complicada } \\
\text { de Corona }\end{array}$} & \multicolumn{1}{c|}{ Procedimientos } \\
\hline Evaluación clinica, radiografia y hallazgos & $\begin{array}{l}\text { Tomar una radiografía periapical, evaluar tamaño de la cámara } \\
\text { pulpar y el estado de desarrollo radicular. Realizar las pruebas } \\
\text { de sensibilidad. }\end{array}$ \\
\hline Tratamiento & $\begin{array}{l}\text { En dientes inmaduros, realizar recubrimiento pulpar o } \\
\text { pulpotomia parcial con hidróxido de calcio y estricto sellado. }\end{array}$ \\
\hline Instrucciones al paciente & $\begin{array}{l}\text { Dieta blanda, cepillado después de cada comida con un cepillo } \\
\text { de cerdas suaves, uso de enjuague bucal con clorhexidina al } \\
0.1 \% \text {, dos veces al dia durante } 7 \text { dias. }\end{array}$ \\
\hline Seguimiento & $\begin{array}{l}6-8 \text { semanas } \\
1 \text { Año. }\end{array}$ \\
\hline
\end{tabular}

Tabla 1. Guía de tratamiento para fractura complicada de corona sugerida por la Asociación Internacional de Traumatología Dental 2001.'

La pulpotomia es un procedimiento que implica la remoción de tejido pulpar coronal que ha sufrido cambios inflamatorios o degenerativos, permaneciendo intacto un tejido remanente vital que es cubierto con un agente que promueve la fijación y la reparación del tejido subyacente. La única diferencia entre pulpotomia y recubrimiento pulpar directo es que en la pulpotomía es removido tejido adicional, la profundidad de este tejido es determinada por el juicio clínico. Si se considera que un tejido está inflamado, debe ser removido. ${ }^{14,15}$

El examen clinico es fundamental para determinar la pertinencia del procedimiento, si se presentan signos o sintomas clinicos como dolor, sensibilidad o movilidad, estos procedimientos no deben realizarse..$^{14.15}$

El propósito de este articulo es presentar el procedimiento clinico de reposición de un fragmento dental en un incisivo superior izquierdo con formación radicular incompleta, ocho dias después de presentar una fractura complicada de corona.

\section{REPORTE DE CASO}

Paciente de 7 años de edad, género femenino, sin antecedentes médicos u odontológicos. Asiste al servicio de odontopediatría de las Clínicas Odontológicas de la Universidad Santo Tomás en Floridablanca, ocho dias después de sufrir dos fracturas en las coronas de los incisivos centrales superiores (Figura 1). El segmento de uno de los dientes fracturados se recuperó en el lugar del accidente, se conservó al medio ambiente y fue llevado a la Institución (Figura 2).
En el examen clínico de la paciente, se observó mordedura en la región anterior de brazo derecho y lesiones rojas en el cuello, no relacionadas con el trauma; según la paciente, éstas fueron propinadas por su hermano mayor de 14 años quien frecuentemente manifestaba esta conducta violenta (Figura 3).

Después de realizar la historia clinica completa, se efectuó la valoración pulpar del 11 y 21. Se diagnosticó una Fractura No Complicada de Corona en el 11 y una Fractura Complicada de Corona en el 21, se encontró una mínima exposición pulpar. Las pruebas periapicales y de sensibilidad fueron positivas. Radiográficamente, se observó la formación radicular incompleta de los dientes, se clasificaron en un estadio de calcificación Nolla ocho, es decir, 2/3 de formación radicular (Figura 4). ${ }^{16}$

Se aisló el campo operatorio con tela de caucho y se practicó un recubrimiento directo en el incisivo central superior izquierdo. Se empleó hidróxido de calcio puro (Indental ${ }^{\circledR}$ ), y agua destilada. ${ }^{18}$ Posteriormente, se aplicó Cavalite de la casa KERR ${ }^{\circledR}$ y se fotopolimerizó. Al instante, se retiraron los excesos para optimizar la superficie de adhesión.

Paralelamente, el fragmento dental fue sumergido en hipoclorito de sodio ( $\mathrm{NaOCl}$ al $5.25 \%$ ) durante 5 minutos, posteriormente en alcohol durante 5 minutos y finalmente, en clorhexidina durante 5 minutos (Figura 5). Una vez realizado este procedimiento se fijó a un cono de gutapercha para facilitar su manipulación (Figura 5). Se le realizó una ranura media en la dentina con el fin de dar lugar al Cavalite colocado sobre el diente. 


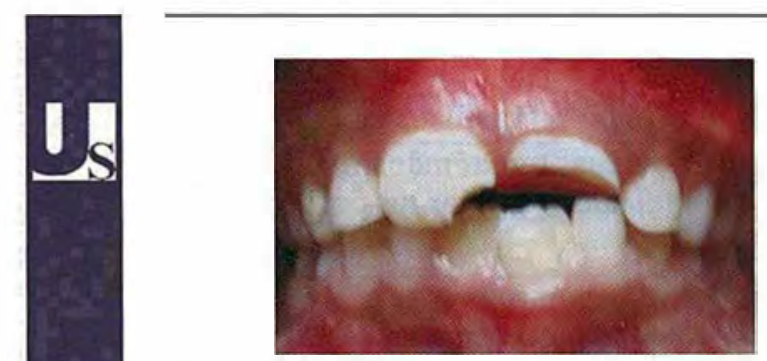

Figura 1. Apariencia clinica de las fracturas de los incisivos centrales superiores.

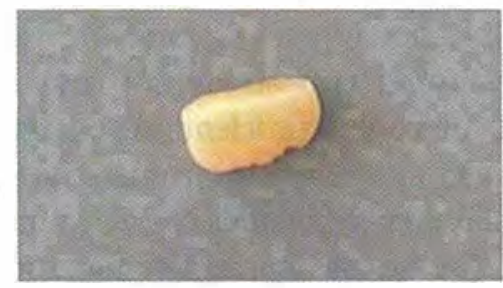

Figura 2. Fragmento recuperado del incisivo central superior izquierdo.

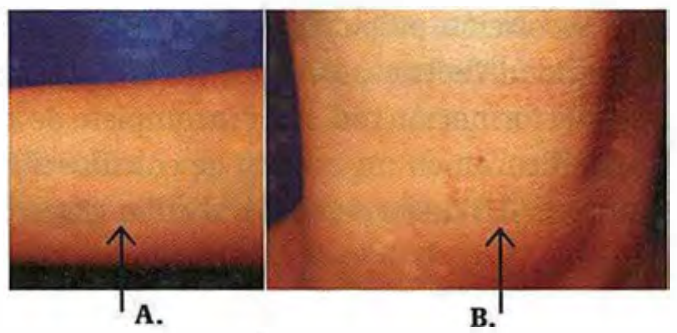

Figura 3. A. Mordedura en región anterior de brazo derecho. B. Extrañas señales en región anterior del cuello.

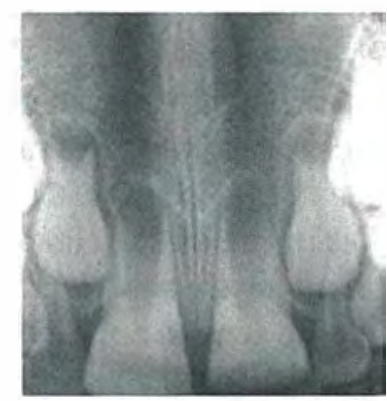

Figura 4. Apariencia radiográfica de los incisivos centrales superiores fracturados.

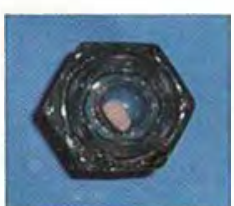

A.

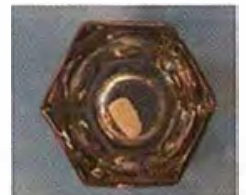

B.

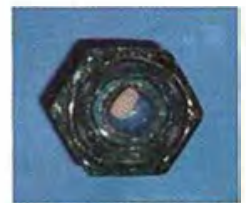

c.
Figura 5. Manejo del fragmento dentario. A. Fragmento sumergido en hipoclorito de sodio. B. Fragmento sumergido en alcohol. C. Fragmento sumergido en clorhexidina.

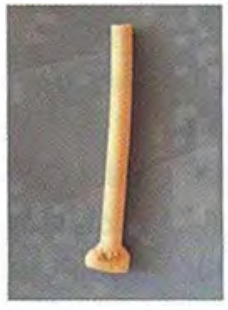

A.

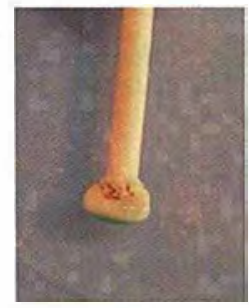

B.
Figura 6. A. Fijación del fragmento dentario a un cono de gutapercha para facilitar su manipulación. B. Grabado del fragmento con ácido fosfórico al 37\% durante 15 segundos.

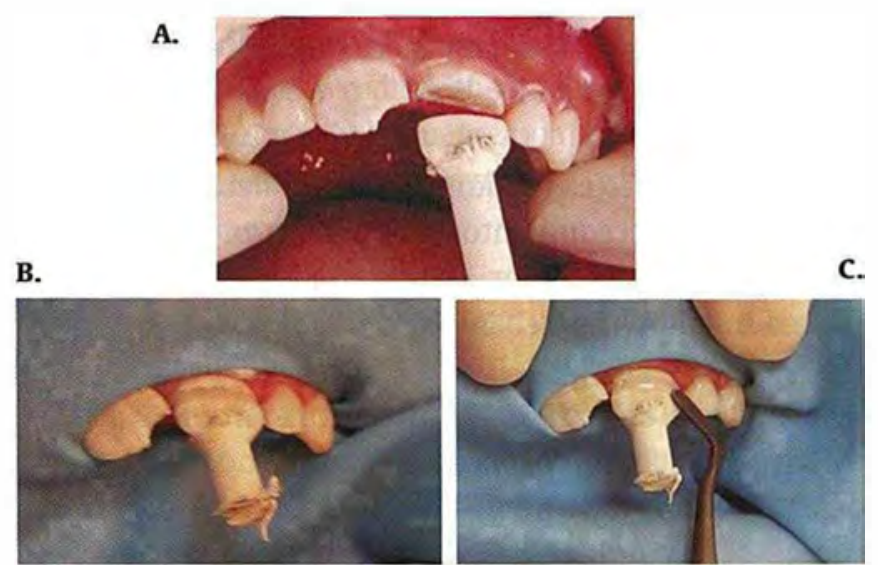

Figura 7. A. Diente con hidróxido de calcio y Cavalite sobre el cuerno pulpar expuesto. B. Fragmento reposicionado en su lugar original. C. Aplicación de resina compuesta sobre la superficie biselada.

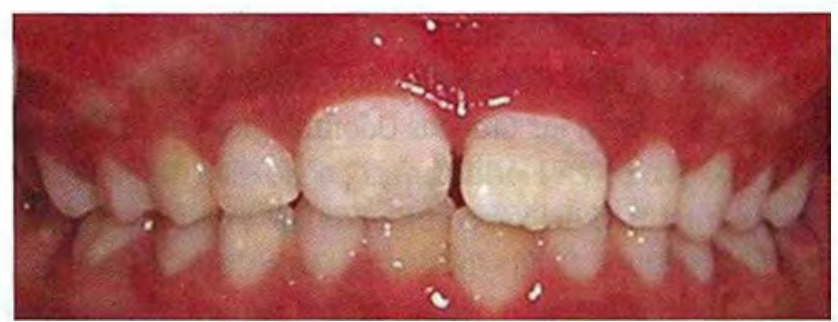

Figura 8. Apariencia clinica del resultado final.

\section{Ee}

Ustasalud Odontología 2005; 4: 59 - 64 


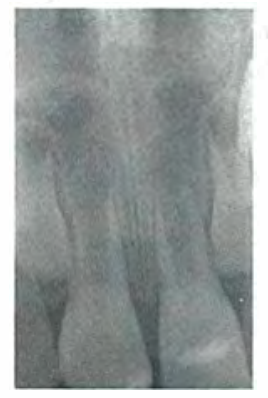

Figura 8. Radiografia final.

Tanto el segmento fracturado como el diente, se grabaron mediante la aplicación de ácido fosfórico al 37\% durante 15 segundos (Figura 6). A continuación, se realizó un biselado con fresa bicóncava para evitar microfracturas y/o desprendimiento de las varillas adamantinas de los bordes del esmalte por la contracción de polimerización de la resina, y por ende desadaptación, filtración marginal y pigmentaciones interfásicas subsecuentes. ${ }^{12}$

Luego se aplicó el sistema adhesivo Single Bond de la casa $3 \mathrm{M}^{\circledR}$ y la resina $\mathrm{P}$, sobre las superficies grabadas. El fragmento fue posicionado exactamente en su lugar original, fotopolimerizando por 40 segundos (Figura 7). Simultáneamente, se realizó el tratamiento restaurador del incisivo central derecho.

Las superficies restauradas fueron pulidas con discos para resinas, montados en pieza de baja velocidad NSK. Se realizó el control de la oclusión. Los resultados muestran una apariencia estética satisfactoria y restauración de la función (Figura 8). En la Figura 9 se observa la apariencia radiográfica final.

Se planean controles radiográficos y pruebas de vitalidad a las tres semanas, tres, seis y doce meses.

\section{DISCUSIÓN}

La reposición inmediata del fragmento es un tratamiento muy conservador que permite la restauración de la anatomía dental original así como la rehabilitación funcional y estética en corto tiempo, por preservación de tejidos dentales, lo que satisface las expectativas del odontólogo y del paciente.
En la actualidad, el mejoramiento de los materiales estéticos nos da la oportunidad de lograr resultados excelentes en la restauración de dientes fracturados. ${ }^{18}$

Aunque la respuesta a nivel microscópico de este procedimiento no ha sido bien estudiada, se ha demostrado éxito cuando la restauración con resina provee un selle óptimo que excluye el paso de bacterias, lo cual es indispensable para la reparación pulpar. $2,4,10.13,19$

Por lo general el recubrimiento pulpar directo se efectúa inmediatamente después de haber sufrido el trauma y cuando la exposición es mínima; en este caso se llevó a cabo 8 días después. En la elección de este tratamiento conservador se tuvieron en cuenta los siguientes aspectos significativos:

\section{Preservación de la integridad pulpar}

Se debe intentar mantener la integridad de la pulpa en los dientes con ápices incompletos con el fin de lograr el desarrollo radicular, de lo contrario puede producirse una raíz delgada y débil propensa a la fractura. El recubrimiento pulpar directo es exitoso en estos dientes debido a que tienen un excelente suministro sanguíneo disponible $a$ través de sus ápices abiertos. ${ }^{15}$

\section{Tipo de trauma dental}

Se trató de una lesión directa que provocó la fractura complicada de la corona y que, evidentemente no lesionó los tejidos de soporte por lo que el pronóstico es mejor, ya que no se afectó el sistema circulatorio de la pulpa y no se vio comprometido su potencial reparativo de este tejido., ${ }^{6,13,20}$

\section{Estado de desarrollo radicular}

La literatura reporta un éxito del $100 \%$ en dientes con fracturas complicadas de corona tratados mediante recubrimiento pulpar directo; se ha observado una clara correlación entre el estado de desarrollo radicular incompleto y el éxito del tratamiento. ${ }^{21}$ Se ha demostrado que en los casos de dientes con formación radicular completa, se favorece la invasión bacteriana y por lo tanto se impide la revascularización pulpar. De esta manera, el diente con ápice abierto tiene un pronóstico más favorable que el diente con ápice cerrado. ${ }^{10}$

\section{Salud pulpar previa}

Se ha estimado como un factor relevante para la supervivencia pulpar por su mayor capacidad reparativa. ${ }^{13}$ 


\section{Elementos estructurales de la pulpa}

Se trata de un órgano joven, altamente celular, con pocas fibras y un rico suministro sanguineo lo cual le otorga una mayor capacidad para elaborar y reparar tejido calcificado y mantener su vitalidad. ${ }^{22}$

\section{Valoración clínica}

A través de las pruebas de sensibilidad, periapicales, examen radiográfico y de acuerdo al juicio clínico se percibió una respuesta pulpar positiva, indicio de una pulpa vital asintomática sin inflamación.

Se determinó el monitoreo continuo de estos dientes traumatizados, mediante pruebas de sensibilidad pulpar, percusión y radiografias, de acuerdo a lo estipulado en las guias de tratamiento para fractura complicada de corona sugeridas por la Asociación Internacional de Traumatología Dental, 2001. ${ }^{11,15}$

En relación con las lesiones presentes en antebrazo derecho y cuello, se realizaron observaciones pertinentes a la mamá de la paciente con respecto a lo que es el maltrato infantil y sus repercusiones legales. En caso de recurrencia se informará a las autoridades competentes.?

\section{BIBLIOGRAFIA}

1. República de Colombia, Ministerio de Salud. Tercer Estudio Nacional de Salud Bucal, ENSAB III, Bogotá: Tomo VIIl, 1998. II Estudio nacional de factores de riesgo de enfermedades crónicas - ENFREC II, 1999

2. Welburv RR. Traumatic injuries to the teeth. Pediatric Dentistry. Third Edition, 2005.

3. Douglass AB, Douglass JM. Common dental emergencies. Am Fam Physician 2003; 67: 511 - 516 .

4. Slack GL. Jones JM. Psychological effect of fractured incisors. Br Dent J 1955; 99: 386 - 388.

5. León JC, Contreras E. Pineda L, Galeano CF. Prevalencia del trauma dentoalveolar en pacientes atendidos en el Servicio de Urgencias de la Clínica Carlos Ardila Lulle de Floridablanca, Colombia, entre 1998 y 2002. Ustasalud Odontología 2004; 3: 32 - 40.

6. Feiglin B. Dental pulp response to traumatic injuries - a retrospective analysis with case reports. Endod Dent Traumatol 1996; 12: 1 - 8.

7. República de Colombia. Ministerio de Salud. Resolución 00412. Guía de atención del menor maltratado. Bogotá, 2000.

8. Hermida MG. El niño con el cual trabajamos. Revista de la Academia Colombiana de Odontología Pediátrica 1998; 2: 17 - 20.
9. Andreasen JO, Andreasen FM. Classification, etiology and epidemiology. Textbook and color atlas of traumatic injuries to teeth. Copenhagen: Munksgaard, 1994: $151-180$.

10. Pinkham JR. Odontología pediátrica. 2da. Edición. McGraw-Hill Interamericana, 1994.

11. Flores MT, Andreasen JO, Bakland LK, Guidelines for the evaluation and management of traumatic injuries. Dent Traumatol 2001; 17: 97 -102 .

12. Uribe EJ. Operatoria dental: Ciencia y práctica. Ediciones Avances, 1995.

13. Andreasen FM. Biology of dental trauma: Pulpal considerations. Procedings, $10-14$.

14. Fucks A. Pulp therapy for the primary and young permanent dentitions. Dent Clin North Am 2000; 44: 571 - 596.

15. American Academy of Pediatric Dentistry. Guideline on pulp therapy for primary and young permanent teeth. Pediatr Dent Reference Manual 2002 - 2003: 24: 86 - 90.

16. Nolla CM. The development of the permanent teeth. J Dent Child 1960; 27: $254-266$.

17. Turner C, Courts F, Stanley HR. A histological comparison of direct pulp caping agents in primary canines. J Dent Child 1987; 54: 423 428.

18. Rapelli G, Massaccesi C, Putignano A. Clinical procedures for the inmediate reattachment of tooth fragment. Dent Traumatol 2002; 18 : $281-284$.

19. Robertson A, Andreasen FM, Bergenholtz G, Andreasen JO, Munksgaard C. Pulp reactions to restorations of experimentally induces crown fractures. J Dent 1998; 26: $409-416$.

20. Robertson A, Andreasen FM, Bergenholtz G. Andreasen JO, Noren JG. Long-term prognosis of crown fractured permanent incisors. The effect of stage of root development and associated luxation injury. Int J Paediatr Dent 2000; 10: 191 - 199.

21. Ravn JJ. Follow-up study of permanent incisor with complicated crown fractures after acute trauma. Scand J Dent Res 1981; 89: 355 $-365$.

22. Van Hazle HJ. Physiology of the human dental pulp. Oral Surg Oral Med Oral Pathol 1971: 32: 126 - 134 